\title{
Obstructive Sleep Apnea: Efficacy Test of the Intraoral Mandibular Advancement Devices by Video Nasopharyngolaryngoscope
}

\author{
Araújo, S.S1; Trindade, M.O2; Arruda, F.E.P3; Fernandes, G.A4
}

\author{
1,3,4Student of the Dentistry Course - UFPE; \\ 2Professor of Prosthesis and Oral and Maxillofacial Surgery Department- UFPE
}

\begin{abstract}
Introduction: Obstructive sleep apnea is a multifactorial case that may involve craniofacial development, bone structures, muscular structures and any soft tissue adjacent to the pharynx have importance to this disorder. It is characterized by recurrent episodes of upper airway obstruction. In Brazil the prevalence is $10 \%$ to $17 \%$ of the population. Recognized as a risk factor for systemic arterial hypertension, stroke and coronary heart disease. For diagnosis, the main exam is the polysomnography. For the evaluation of the efficacy of the therapies, such as the intraoral device, different exams have been proposed in the literature: computed tomography in sagittal, parasagittal, coronal sections, $3 \mathrm{D}$ reconstructions, cephalometry and nasolaringopharyngoscopy video. Objective: The aim of this paper is provide to professionals who work with sleep apnea, a less invasive solution to prove the efficacy of the therapies used. Methodology: The paper is a literature review to evaluate the results of nasolaringopharyngoscopy in patients undergoing treatment with intraoral device for sleep apnea without and during the use of the device to evaluate the opening of the larynx and pharynx. During the wake-up examination, the patient should undergo the Müller's maneuver, which consists of deep breathing and maintaining inspiration by obstructing the upper airways, with room for the instrument only. Results and Discussion: It demonstrated that it is possible to do the examination, even if the patient is with the device. There is pronounced distinctness in this exam, which allows to accurately evaluate the clearance with or without devices, if it is well adapted. As a disadvantage, airway measurements are not accurate as on a CT scan. Conclusion: The nasolaringopharyngoscopy video is effective for qualitative analysis of the device use, but does not support in a quantitative analysis on the airways.

*Correspondence to Author:

Araújo, S.S

Student of the Dentistry Course UFPE

How to cite this article:

Araújo, S.S; Trindade, M.O; Arruda, F.E.P; Fernandes, G.A.Obstructive Sleep Apnea: Efficacy Test of the Intraoral Mandibular Advancement Devices by Video Nasopharyngolaryngoscope.International Research Journal of Otolaryngology, 2018, $1: 1$

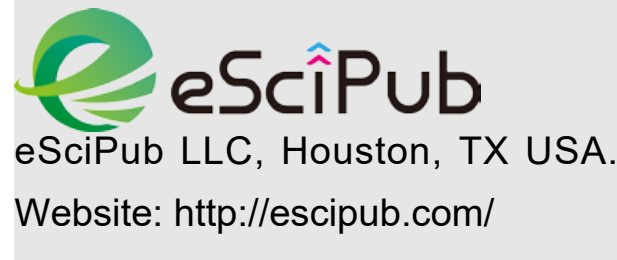

Keyword: Airway obstruction; Pharynx; Sleep apnea syndromes 